$$
\text { DOE/OR/D0033-T65T }
$$

Pat

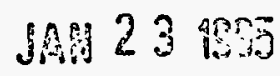

OSTI

FEATURE DETECTION FOR SPATIAL TEMPLATES

BY

KAREN ROBINSON

MORGAN STATE UNIVERSITY

DISCLAIMER

This report was prepared as an account of work sponsored by an agency of the United States Government. Neither the United States Government nor any agency thereof, nor any of their employees, makes any warranty, express or implied, or assumes any legal liability or responsibility for the accuracy, completeness, or usefulness of any information, apparatus, product, or process disclosed, or represents that its use would not infringe privately owned rights. Reference herein to any specific commercial product, process, or service by trade name, trademark, manufacturer, or otherwise does not necessarily constitute or imply its endorsement, recommendation, or favoring by the United States Government or any agency thereof. The views and opinions of authors expressed herein do not necessarily state or reflect those of the United States Government or any agency thereof.

\title{
MASTER
}

DISTRIBUTION OF THIS DOCUMENT IS UNLIMITED 


\section{FEATURE DETECTION FOR SPATIAL TEMPLATES}

\subsection{Executive Summary}

The Color Medical Image System (CMIS), a program that uses segmented mapping techniques to obtain high resolution digital images, is currently trying to develop techniques to transfer microscopic glass slides to electronic image libraries. One technique that has been attempted is to use correlation techniques to scan the image. However, when segments of high magnification are used, it is difficult and time consuming to perform correlation techniques. This project investigates feature detection in microscopic images. Various techniques are implemented to detect the section of the image containing the most feature information, thereby making the correlation process more efficient.

Three tests are implemented that eliminate the background in the image and calculate the mean (1st order technique), variance (2nd order technique), and ratio test (1st order technique) of the remaining pixel values. Background elimination involves deleting all pixel values above a certain experimental value from any calculations made. The source code for each of the three tests was implemented and tested on a number of images using the green color band. Each program outputs the box containing the most features and writes that section to a file to be displayed to the screen. A visual rank was also recorded so as to compare it the output of the tests.

Each of the three tests proved to be successful. After comparing the visual rank to the output of the tests, it was determined that both first and second order techniques are effective in detecting features in microscopic images. Although all of the purposes and goals were met, this investigation should be expanded to include texturized images and the use of all three color bands.

\subsection{Introduction}

The Color Medical Image System (CMIS)) program uses segmented capture and mapping techniques to obtain high resolution digital images of microscopic biomedical images from multiple digitized video images. The goal of CMIS is to develop techniques to transfer existing clinical microscopic glass slide collections to electronic image libraries.

When scanning an image to be stored, a series of overlapping segments must be taken to ensure that all important information, including the edges of the image, will be obtained. Once these segments are taken, they must be superimposed so as to capture the complete, original image. A process called correlation can be used to accomplish this by sliding two signals into one another and determining where the correlation equation will produce its maximum product. One of the principle applications of correlation is in the area of template matching, where the problem is to find the closest match between an unknown image and a set of known images. 
The problem arises when segments of high magnification such as microscopic images, are used. In segments at high magnification, there can be gaps in the image features. Because of these gaps in features, it is difficult as well as time consuming to perform correlation techniques. This particular project investigates feature detection for spatial templates. The purpose of this project is to develop and evaluate techniques which detect spatial properties of image features. This investigation will test, compare, and analyze the effectiveness of first and second order techniques in detecting image feature for template matching. Various techniques will be implemented to detect the section of the image containing the most feature information, thereby making correlation more efficient.

\subsection{Tools}

The tools used in developing this project is as follows:

\subsection{Developed Algorithms}

The algorithms developed for this project are written in $\mathrm{C}$ language and can be found in the appendix. It incorporates varied and intricate loops to implement each of the three hypotheses.

\subsection{Histogram Software}

A histogram plot is a graph showing the range of pixel values $(0-255)$ versus the number of times that each pixel value appears in a particular image. This software was extremely helpful in determining and visualizing the effects of the background elimination process (which will be further discussed).

\subsection{Image Display Software}

The image display software outputs an image or a specified section of an image to the user screen. This software was necessary to determine the efficiency of the three tests. It was also useful in recording a visual rank of the section of the image containing the most features.

\subsection{Discussion of Hypotheses}

Given are adjacent images with dimensions 512 pixels by 480 pixels that contain some of the same feature information. A section of an image will be divided into eight equivalent squares numbered 0-7, each having dimensions of 90 pixels by 90 pixels. A series of tests will be implemented on each of the eight squares to determine which box has the most feature information. Below is a representation of the eight equal divisions in a given image. 


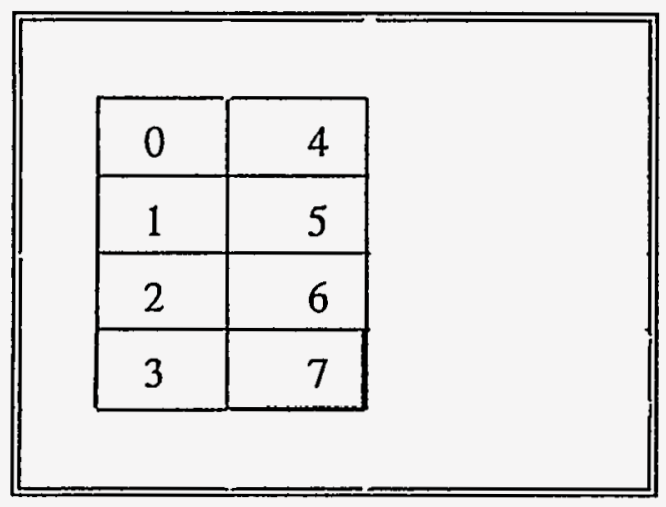

In order to isolate the feature information, each of the following three test involves background elimination. This means that all pixel values above a certain experimental value will be deleted from any calculations made. Since most background information is usually lighter than the feature information, the experimental pixel value should be in the higher gray level value range.

\subsection{Background to Foreground Ratio Selection \{1st order technique\}}

This test will eliminate the background of a given image and perform a ratio test of the number of pixels remaining divided by the total number of pixels. The section having the greatest percentage should contain the most features.

The program first sets all the background to 255 (white). It then finds the section of the image that has the greatest ratio. The program also prints the calculated ratio for each box. Since each box is assigned a numerical value ranging from $0-7$, the number of the square containing the most feature information is printed to the screen.

If a situation occurs where the ratio of two different boxes is equal, then the program will keep the first square found as having the most ifeatures. This technique is acceptable since the primary purpose of this project is to find an area of an image with substantial $f$ e a $t u r e$ information; if there occurs more than one section with a lot of features, then any one of those sections will suffice.

\subsection{Distance of the Means \{1st order technique\}}

This test will calculate the mean of the histogram with and without the presence of background. The area that has the least shift of the mean should contain the most features. Below is the equation that is used to calculate the mean

$$
U=1 / N \sum \mathbf{I}_{\mathbf{i}}
$$


where $\mathbf{U}$ is the mean value, $\mathbf{N}$ is the total number of pixels in the image, and $\mathbf{I}_{\mathbf{i}}$ is the ith pixel of the image.

The mean of a group of numbers is, in essence, the average. Therefore, the average of each of the pixel values in a particular section is taken. The average is taken once again after the background is eliminated and the two averages are subtracted. This value represents the shift of the mean. Therefore, if a section of an image contains a lot of features and very little background, the shift of the mean will be quite small. In other words, the two mean values will be approximately equal because the elimination of background did not greatly affect the image. The least shift of the mean will determine which one of the eight squares contain the most feature information. As in the first hypothesis, the mean of each square will printed to the screen. The program also outputs the square that has the most features.

\subsection{Optimum Variance \{2nd order technique\}}

This test will calculate the variance of the histogram with and without the background. Through experimentation, the area with the optimum variance should contain the most feature information. Below is the equation that is used to calculate the variance.

$$
\operatorname{VAR}=1 / N \sum\left(I_{i}-U\right)^{2}
$$

As in the hypothesis concerning the distance of the means, the variance of the histogram of each square was calculated with the background present and calculated once more without the presence of background. These two values were subtracted and it was experimentally discovered that the square that had the least shift of the variance should contain the most feature information. The calculated variance of each square is printed to the screen as is the square that has the most feature information. The image display software could be used for each of the three hypotheses to actually see the section of the image chosen by the program as having the most features.

\subsection{Data Collection}

Using the green color band, a number of microscopic images were used to test each of the three programs. For each image, a histogram plot was graphed before and after the elimination of the background. A comparison of the two gives a clear representation as to the alterations done to the pixels in the image after the background elimination.

After successfully compiling each test, the image display software was implemented and the section of the image containing the most features was displayed to the screen. A visual rank of each square in the image was recorded using a scale of good, fair or poor. This rank was implemented so as to compare the effectiveness of the three programs with the human eye. 
Below is chart of the ranking of some of the images used.

\begin{tabular}{||l|c|c|c|c|c|c||}
\hline \hline IMAGE & & & & & & \\
\hline & Test 1 & Test 2 & Test 3 & \multicolumn{1}{|c|}{ Good } & \multicolumn{1}{|c|}{ Fair } & Poor \\
\hline 66fe03.g58 & 0 & 0 & 0 & $\begin{array}{l}* 0, * 1, \\
* 3,4,5, \\
* 6, * 7\end{array}$ & \multicolumn{1}{|c||}{} & --- \\
\hline 67fe03.g58 & 3 & 3 & 3 & $* 3,7$ & 6,0 & $1,2,4,5$ \\
\hline 68fe03.g58 & 7 & 7 & 7 & 3,7 & 2,6 & $0,1,4,5$ \\
\hline 41pam101.g54 & 5 & 5 & 5 & 7 & $1,2,6$ & $0,3,4,5$ \\
\hline 42pam101.g54 & 2 & 2 & 2 & $2,4,5$ & $1,3,6,7$ & 0 \\
\hline 43pam101.g54 & 1 & 1 & 1 & 1,3 & $0,4,5,7$ & 2,6 \\
\hline 11pam101.g52 & 2 & 2 & 2 & $* 2,6$ & $\begin{array}{l}0,1,4,5, \\
7\end{array}$ & 3 \\
\hline 12pam101.g52 & 6 & 6 & 6 & $0,3,4$, & $1,2,6,7$ & - \\
\hline 13pam101.g52 & 1 & 1 & 1 & $* 2, * 5,1$ & $0,3,4,6$, & 7 \\
\hline center8x.g51 & 5 & 1 & 5 & $* 1,6$ & $0,2,4,5$ & 3,7 \\
\hline fdq03.g51 & 1 & 1 & 1 & 1 & $3,5,7$ & $0,2,4,6$ \\
\hline
\end{tabular}

NOTE: * means the square(s) with the most feature information.

As the chart explains, each of the three tests chose the same section of the image as having the most feature information. For one particular image (center8x.g51), however, the Distance of the Mean Test chose a different square as containing the most features. When the visual rank was accessed, it was seen that the Distance of the Mean Test had chosen the correct square while the other two tests chose the incorrect section of the image. Both the Background to Foreground Ratio Selection Test and the Optimum Variance Test chose a square that had a fair amount of feature information. This discrepency may have been attributable to visual rank. This ranking system was rather difficult to categorize since some of the images either contained alot of feature information or alot of background. 


\subsection{Discussion of Results}

Overall, each of the three tests effectively chooses the square containing the most feature information. When the results of the tests were compared to the visual rank, it was verified that the square chosen from the test did, in fact, contain the most features. When implementing the visual rank, some images had more than one square that had a lot of feature information. When this occurs, all of the squares containing a lot of feature information is recorded. The tests would always output one of the squares recorded in the visual rank as having good feature information.

With the exception of one image, all three test gave the exact output. As discussed previously, the Distance of the Mean Test chose the correct section of the image while the other two did not. Therefore, it can be said that the hypothesis concerning the distance of the means was slightly more efficient than the other two hypotheses. However, it can be said that each test was extremely proficient in detecting the section of the image with the most feature information.

This project was originally supposed to be incorporated with a correlator which would actually map the two adjacent segments back together using the section of the image chosen by one of the three hypotheses. However, due to lack of time and resources, this section of the project had to be eliminated.

\subsection{Summary/Conclusion}

This project was extremely successful. The goals and objectives of this investigation were met. Three tests were developed that detected spatial properties of image features. Each of the three test successfully chose the section of the image containing the most features. The Distance of the Means Test was more proficient than the other two tests and therefore can be said to be the most efficient of the three tests. However, both first and second order techniques prove to be effective in solving this problem. The scope of this project can be broadened to include texturized images and also images in all three color bands.

Feature Detection For Spatial Templates was an extremely interesting investigation that exposed me to the independent research environment required in graduate school. This project also helped me to understand C-language as it relates to current problems in the world. The Color Medical Image System can use a combination of the techniques used in this project to develop techiques to transfer microscopic glass slides to electronic image libraries. 


\subsection{Works Cited}

Gonzalez, Pafael C and Richard E. Woods. Digital Image Processing. Addison-Wesley Publishing Company: Reading, 1992.

Myler, Harley R. and Arthur R. Weeks. Computer Imaging Recipes in C. PTR Prentice Hall: Englewood Cliffs, 1993.

Waite, Mitchell and and Don Martin. The Waite Group's UNIX System V Primer. Prentice Hall Computer Publishing, Carmel, 1992.

Waite, Mitchell and Stephen Prata. The Waite Group's New C Primer Plus. Carmel, 1990. 